\title{
A metallic hot-carrier photovoltaic device
}

\author{
James A. R. Dimmock ${ }^{* 1}$, Matthias Kauer ${ }^{2}$, Jiang $\mathbf{W u}^{3}$, Huiyun Liư ${ }^{3}$, Paul N. Stavrinou ${ }^{4}$, Nicholas J. Ekins- \\ Daukes $^{5}$
}

\author{
${ }^{1}$ Sharp Laboratories of Europe Ltd, Oxford, United Kingdom \\ ${ }^{2}$ Lightricity Ltd, Oxford, United Kingdom \\ ${ }^{3}$ University College London, London, United Kingdom \\ ${ }^{4}$ University of Oxford, Oxford, United Kingdom \\ ${ }^{5}$ University of New South Wales, Sydney, Australia
}

E-mail: dimmock@cantab.net

\begin{abstract}
Hot carrier solar cells overcome the fundamental limitations of conventional devices where charge carriers are photogenerated over a broad energy spectrum but rapidly lose energy to the lattice and are extracted at the lowest energy of the system. In a hot carrier photovoltaic cell, carriers are extracted at higher energies than the absorption threshold, and extraction proceeds sufficiently quickly to avoid dissipative energy loss. We demonstrate a new hot carrier photovoltaic device where a broad spectrum of light is absorbed in metallic layers tens of nanometers thick. Using a semiconductor quantum well resonant tunnel structure we demonstrate energy selective hot carrier extraction from the metal film and show a unique hot carrier signature in the device IV characteristics. Using a multiple beam experiment, we further prove that these carriers arise from a hot electron population rather than via internal photoemission; a necessary requirement for high efficiency photovoltaic operation.
\end{abstract}

Keywords: hot carrier, photovoltaic, solar cell, metal absorption

\section{Introduction}

Conventional photovoltaic cells are fundamentally limited in their efficiency because a broad spectrum of light is used to drive a two-level system. Charge carriers are generated with a broad spectrum of energies, but rapidly dissipate energy to the lattice and are extracted at lower energies, the valence and conduction band edges. By contrast hot carrier solar cells operate by maintaining electrons in two regions of the cell at different temperatures, forming a heat engine by allowing selective extraction of electrons from the hotter region to the colder region, with a limiting efficiency for such a system of $85 \%{ }^{1}$. Recently, promising initial results, proofs of principle and materials developments ${ }^{2-10}$ have shown the potential of this concept. Here we demonstrate the first broadband hot carrier device.

One challenge present in conventional solar cells, but particularly acute in hot carrier cells, is simultaneously achieving high, broadband absorption and optimum extraction. The extraction length scale for a hot carrier cell is significantly shorter than for a conventional solar cell, as extraction of electrons needs to occur prior to electron thermalisation, a process which often occurs on a timescale of picoseconds ${ }^{11}$. Counter-intuitively, it is also necessary that extraction of electrons not occur too quickly, as is the case with structures such as the Internal Photoemission (IPE) cell ${ }^{12-16}$. If electrons are extracted before they equilibrate into a thermal population, i.e. they are extracted as ballistic electrons, no efficiency gain is obtained from the redistribution of carrier energy. As such, relative timescales of carrier thermalisation, equilibration and extraction are crucial in the hot carrier cell ${ }^{17,18}$, if we are to achieve a properly functioning device.

We demonstrate a hot carrier solar cell structure in which absorption of light occurs in a thin metal layer, taking advantage of the very high extinction coefficient present in metals. Extraction and exploitation of the absorbed energy depend on the mechanism of absorption and the resulting excited carriers.
For metals, there are four general light absorption mechanisms ${ }^{19}$ : interband absorption and three types of intraband absorption, in which momentum is conserved by either phonon emission, electron-electron scattering or surface plasmon-polariton generation ${ }^{20}$. In the optical and NIR wavelengths investigated in this work and dominating the solar spectrum, the metal that we use $(\mathrm{Cr})$ shows little interband absorption, and is not deliberately structured or illuminated to encourage the generation of surface plasmonpolaritons ${ }^{21}$. Therefore, direct absorption, with momentum conservation through either electron-electron scattering or phonon emission, are the key absorption pathways. This mechanism results in the generation of a population of electrons with a temperature greater than the lattice temperature.

To exploit the heated electron population, a thin metal layer may be formed on the surface of a semiconductor, with two different heterostructures, providing two different modes of electron extraction. In one device, the metal layer is formed on an n-doped semiconductor, forming a Schottky barrier, over which excited electrons can be extracted by thermionic emission (Figure 1a). In a second device, the metal layer is formed on an n-doped, graded quantum well, allowing extraction of electrons by tunneling (Figure 1b). These two mechanisms provide both different timescales of extraction (with thermionic emission being orders of magnitude faster than tunneling), and also different energetic selectivity (with thermionic emission providing a high-pass energy filter, and tunneling producing a notch filter in terms of the energy of electrons that are permited to flow). These different modes of extraction, and their difference to the IPE cell (Figure 1c), present themselves as distinct differences in the IV characteristics under illumination, which are discussed and demonstrated in this work. 

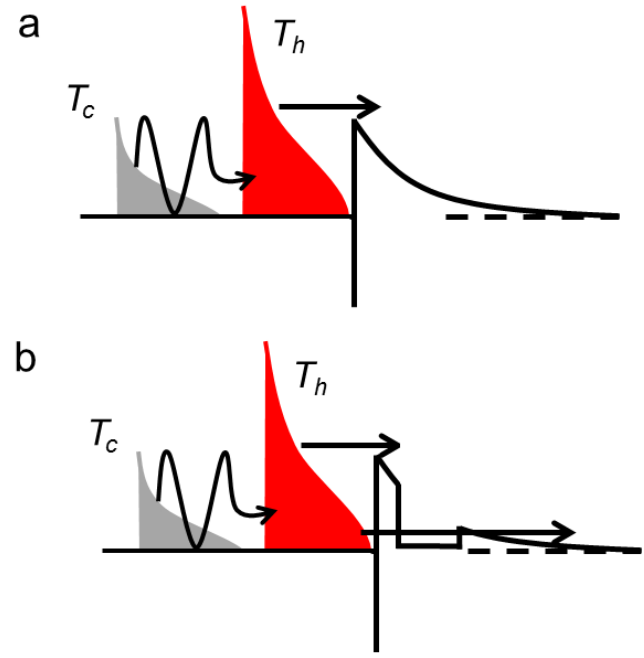

C

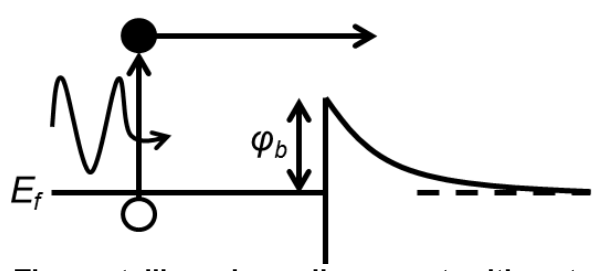

Figure 1 The metallic solar cell concept with extraction by thermionic emission (a) and tunneling (b) in contrast to an internal photo-emission (IPE) cell (c). Showing the heating of a population of electrons initially at temperature $T_{c}$ and subsequently at temperature $T_{h}$ upon absorption of light, followed by extraction of electrons from this heated distribution, in contrast with the immediate extraction of a ballistic electron in the IPE cell.

\section{Devices and Experimental Methods}

Three devices are fabricated and tested to explore the concept of hot carrier extraction from a thin metal film. The first device, SB1, has 8nm of Chromium thermally evaporated onto an n-doped GaAs substrate, the thinnest possible layer of $\mathrm{Cr}$ which could still form a planar layer and not islands. This device is used to establish the general principle of hot carrier extraction following absorption in metal. Of particular interest is whether such structures operate as a hot carrier cell, a Schottky cell or an IPE cell. The study also draws on two further structures, comprising an energy-selective layer, designed to compare the impact of hot-electron extraction via tunneling, These structures have $30 \mathrm{~nm}$ of Chromium deposited on $\mathrm{Al}_{0.4} \mathrm{Ga}_{0.6} \mathrm{As}$, with (QW1) and without (SB2) a graded $\mathrm{Al}_{x} \mathrm{Ga}_{(1-x)}$ As quantum well $4 \mathrm{~nm}$ from the metal layer. The layer structures of the samples are shown in tables 1-3.

\section{Sample Growth and Preparation}

The heterostructures were grown by Molecular Beam Epitaxy on GaAs substrates. After oxide desorption of GaAs substrates, a $500 \mathrm{~nm}$ GaAs buffer layer was grown. The heterostructures, were then grown on the GaAs buffer. The graded $\mathrm{Al}_{\mathrm{x}} \mathrm{Ga}_{1-\mathrm{x}} \mathrm{As}(\mathrm{x}=0-0.3)$ well was grown by the digital alloy method. The GaAs epilayers were grown at $580{ }^{\circ} \mathrm{C}$ and AlGaAs epilayers were grown at $600{ }^{\circ} \mathrm{C}$. Both samples were grown with a $20 \mathrm{~nm}$ GaAs capping layer to prevent oxidation of AlGaAs. This was etched with a selective citric acid based etch immediately prior to loading into an Edwards Auto 306 thermal evaporator, where films of Chromium were deposited at $0.01 \mathrm{~nm} / \mathrm{s}$ at a base pressure of $4 \times 10^{-6} \mathrm{mbar}$.
Table 1 | The processed layer structure of SB1

\begin{tabular}{|c|c|c|}
\hline Layer & Material & Thickness [nm] \\
\hline Absorber & Chromium & $8 \mathrm{~nm}$ \\
\hline Collector & $\begin{array}{c}\mathrm{nGaAs} \\
1.8 \times 10^{17}(\mathrm{Si})\end{array}$ & Substrate (300um) \\
\hline
\end{tabular}

Table 2 | The processed layer structure of QW1

\begin{tabular}{|c|c|c|}
\hline Layer & Material & Thickness [nm] \\
\hline Absorber & Chromium & $30 \mathrm{~nm}$ \\
\hline Barrier & $\begin{array}{c}\mathrm{Al}_{0.4 \mathrm{Ga} 0.6 \mathrm{As}} \\
5 \times 10^{17}(\mathrm{Si})\end{array}$ & $4 \mathrm{~nm}$ \\
\hline Well & $\begin{array}{c}\mathrm{Al}_{\mathrm{x}} \mathrm{Ga}_{(1-\mathrm{x})} \mathrm{As}: \\
\mathrm{x}=0 \rightarrow 0.3 \\
5 \mathrm{x} 10^{17}(\mathrm{Si})\end{array}$ & $15 \mathrm{~nm}$ \\
\hline Collector & $\begin{array}{c}\mathrm{Al}_{0.4 \mathrm{Ga} 0.6 \mathrm{As}} \\
5 \times 10^{17}(\mathrm{Si})\end{array}$ & $150 \mathrm{~nm}$ \\
\hline
\end{tabular}

Table 3 | The processed layer structure of SB2

\begin{tabular}{|c|c|c|}
\hline Layer & Material & Thickness [nm] \\
\hline Absorber & Chromium & $30 \mathrm{~nm}$ \\
\hline Collector & $\begin{array}{c}\mathrm{Al} l_{0.4} \mathrm{Ga} 0.6 \mathrm{As} \\
5 \times 10^{17}(\mathrm{Si})\end{array}$ & $169 \mathrm{~nm}$ \\
\hline
\end{tabular}

The dark current as a function of temperature was taken for all cells after initial fabrication and also approximately one month post-fabrication. Measuring the dark current as a function of temperature allows an estimation of the Schottky barrier height ${ }^{22}$. In addition to revealing this important device characteristic, any changes observed in the dark current after one month of experimentation reveal important information about device stability and therefore the reliability of results. All devices presented suffer an RMS current difference of less than $5 \%$ over the range $-0.5 \mathrm{~V} /+0.5 \mathrm{~V}$ at room temperature after one month of testing.

The Cr layer thickness in QW1 and SB2 was non-optimal in terms of its light absorption properties in order to counter the degradation that we observed for cells with an AlGaAs semiconductor region and a thinner $\mathrm{Cr}$ layer. This resulted in a reflectivity for these cells of $65 \%$ at $850 \mathrm{~nm}$, but had the benefit that no light passed into the semiconductor region, irrespective of wavelength, unlike SB1 with the thinner $\mathrm{Cr}$ layer. In addition, by using $\mathrm{Al}_{0.4} \mathrm{Ga}_{0.6} \mathrm{As}$ as the semiconductor material, the band gap is widened by $0.5 \mathrm{eV}$, allowing illumination with shorter wavelengths of light without the concern of absorption in the semiconductor region.

The graded well region for QW1 was designed to have a flat band at operating bias while still maintaining a $0.1 \mathrm{eV}$ barrier to the AlGaAs region at the interface furthest from the metal/semiconductor barrier, as such it has a width of $15 \mathrm{~nm}$ and the Aluminium fraction is graded from 0 to 0.3 in the direction away from the metal layer.

The calculated bandstructure for QW1, using the determined effective Schottky barrier height, is shown in Figure 2. The energy of the confined electron state, through which electron tunneling can occur from the metal, is shown as a blue dashed line, the corresponding hole confined state is shown in red. The bandstructure was calculated using a selfconsistent Schrödinger-Poisson model, described in previous work $^{18}$. 


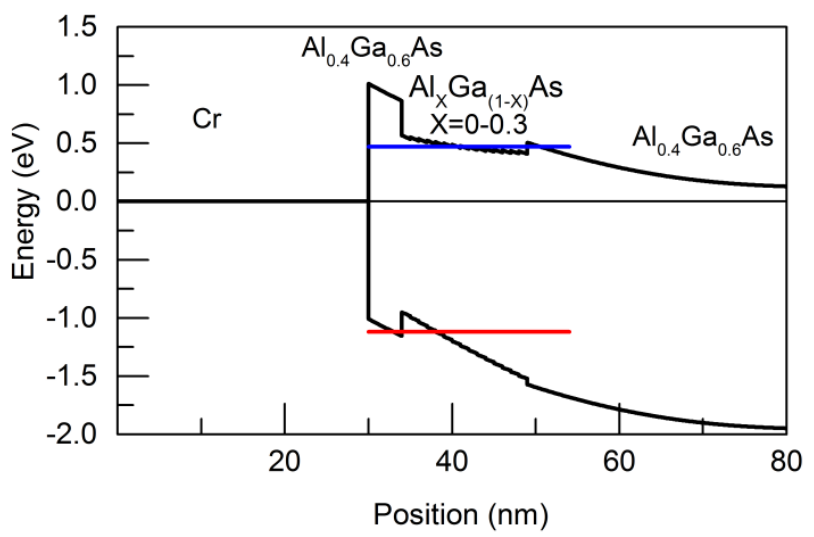

Figure 2| The calculated band structure of the QW1 tunneling structure, showing the Schottky barrier to the Chromium layer

\section{Measurement Details.}

Illuminated IV characteristics were obtained by illuminating the cells with various laser beams focused onto the fabricated cell mesa. A confocal system with an objective lens was used to both image the cell and to focus the light to a spot onto the active region. The sample was mounted on a cryogenic microscope stage (Linkam THMSE600 cryogenic microscope stage) to provide temperature dependent light IV measurements from $80 \mathrm{~K}$ to room temperature. Three different illumination sources were used as an input light source for this confocal system:

A wavelength tuneable (700-950nm) $\mathrm{Ti}: \mathrm{Al}_{2} \mathrm{O}_{3}$ laser with a minimum pulse width of $1.2 \mathrm{ps}$ and an $80 \mathrm{MHz}$ repetition rate. (Spectra-Physics: Millennia pumped Tsunami)

A variety of laser diodes driven by a variable pulse width and duty cycle driver, minimum 100 ns pulse width and $1 \%$ duty cycle up to continuous wave. (ILX Lightwave LDP3840B)

Eight fibre-coupled diode lasers from 642nm-1550nm which can be combined in a wavelength multiplexer to allow simultaneous illumination with up to three separate laser wavelengths on the same focused spot. The power of each fibre-coupled laser can be modulated separately for lock-in measurements. (THORLABS MCLS1 system).

IV characteristics were measured with a Keithley 2400 sourcemeter every $0.01 \mathrm{~V}$ and are presented as line plots, because data points would merge into a line on the scale shown.

The most pronounced effect of the dual wavelength illumination comes at voltages prior to the $\mathrm{V}_{\text {oc }}$ point and with longer wavelengths that excite carriers that only just overcome the Schottky barrier. For this reason, we have compared the response of $\mathrm{QW} 1$ and $\mathrm{SB} 2$ at $-0.2 \mathrm{~V}$ before the $\mathrm{V}_{\text {oc }}$ point over the full temperature range. To reduce the noise present in the subtracted data, the illumination intensity of the $852 \mathrm{~nm}$ beam was modulated to allow lock-in amplification of the related changes in current at this fixed applied voltage.

\section{Schottky Barrier Metallic Solar Cells}

SB1 was illuminated with laser wavelengths of $785 \mathrm{~nm}$, $850 \mathrm{~nm}$ and $890 \mathrm{~nm}$, chosen to allow tuning to either side of the GaAs absorption edge by varying substrate (i.e. lattice) temperature. The beams were focused to provide an intensity of $16 \mathrm{~W} / \mathrm{cm}^{2}$. The resulting IV characteristics are normalised to $\mathrm{J}_{\mathrm{sc}}$ to allow comparison across wavelength and temperature and are presented in Figure 3, for temperatures from 123$263 \mathrm{~K}$ and at the three wavelengths. In addition to the normalised data, the absolute values of $\mathrm{J}_{\mathrm{sc}}$ for the wavelengths and temperatures shown are plotted as Figure $3 d$.

The thickness of Chromium in SB1 results in a semitransparent layer, as would be optimal for placement in a cavity to allow broadband absorption ${ }^{23}$. This has the additional benefit of allowing light to be absorbed in the GaAs region, if it is in excess of the semiconductor band gap. The structure allows the cell to be probed operating in its intended mode of operation, with absorption in the metal, and compare it with the operation as a standard Schottky solar cell with absorption in the GaAs.

Under these two distinct modes of operation, the different nature of electron extraction reveals itself in differences in the IV characteristics, presented in Figure 3.

In a standard n-semiconductor Schottky cell the absorption of light occurs in the semiconductor region. Electrons are driven from the metal $/ \mathrm{n}$-Semiconductor interface into the semiconductor bulk by the field created by the depletion of electrons from this region. Since holes in the valence band experience a field driving them into the metallic region this creates a negative current (electron flow in the opposite direction to potential). As the potential on the metal layer is increased the barrier height is reduced, until the point at which the electrons flow into the metal rather than into the semiconductor. From this point, the current abruptly turns positive as the photogenerated electrons and the dopant electrons in the n-semiconductor now contribute to a large forward biased diode current. This mode of operation is schematically illustrated in Figure 3.

This mechanism of current generation results in two important features of the Schottky solar cell IV. Firstly, a reverse bias current which saturates at the value of the incident photon flux (until the breakdown of the junction at large reverse bias). Secondly, $\mathrm{dI} / \mathrm{dV}$ in forward bias, but before $\mathrm{V}_{\mathrm{oc}}$, determined by shunt resistance and, ideally, zero in a perfect cell. These features are seen for $785 \mathrm{~nm}$ illumination in Figure 3c, a wavelength for which absorption at every temperature occurs in the GaAs region as well as the chromium region. The poor fill factor observed in this device for this wavelength of illumination is due to absorption still occurring in the metal region, thus creating a hot contact, resulting in a high dark current.

Contrast this operation with the case of the metallic cell, where the absorption of light occurs in the metallic region, also schematically shown in Figure 3. The creation of a heated electron distribution in the metal results in carriers being transferred from the metal to the semiconductor. Only carriers with energies in excess of the Schottky barrier height can contribute to the current and as such the current, figuratively, traces out the shape of the electron Fermi distribution as the bias changes. This results in two large differences in the IV characteristics from the Schottky solar cell. Firstly, the reverse bias current does not saturate and secondly $\mathrm{dI} / \mathrm{dV}$ in forward bias, but before $\mathrm{V}_{\mathrm{oc}}$, is determined by the shape of the Fermi distribution even in the case of infinite shunt resistance. 

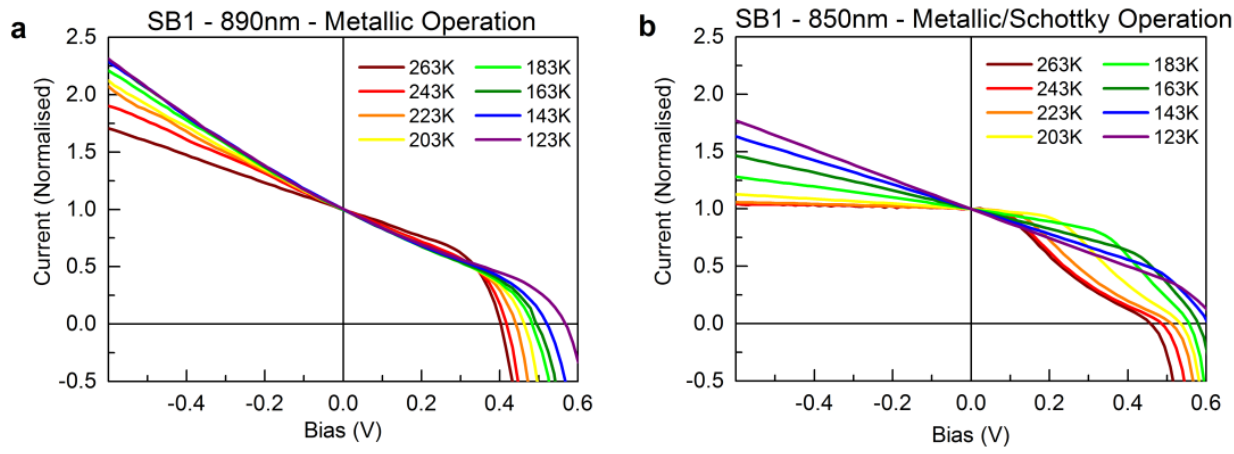

Long Wavelength: Metallic Cell Operation
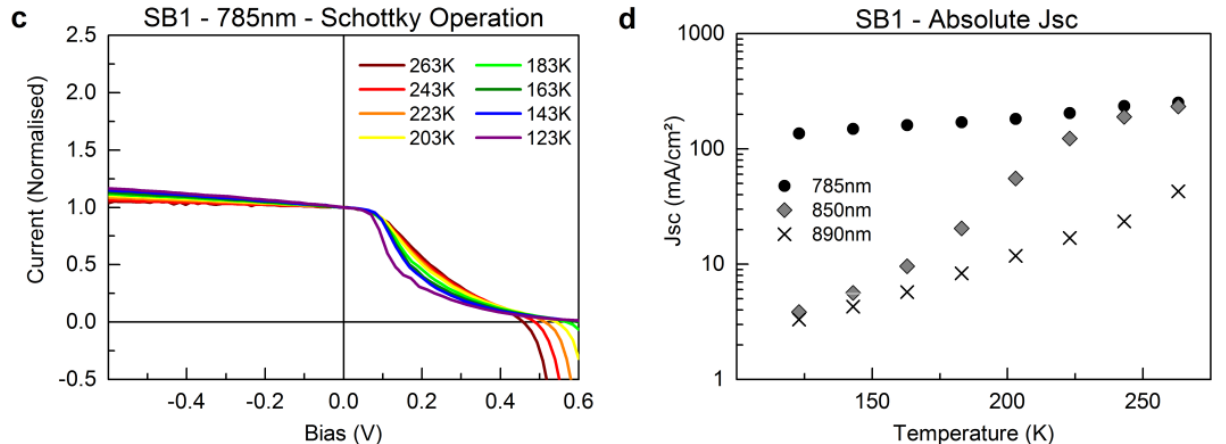

Short wavelength: Schottky Cell Operation Absorption in Semiconductor

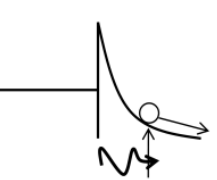

Reverse bias

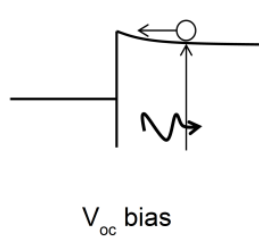

Figure 3 | The absolute normalized IV characteristic of a metallic solar cell (SB1) with thermionic extraction at three different wavelengths: a) $890 \mathrm{~nm}$, b) $850 \mathrm{~nm}$ and c) $785 \mathrm{~nm}$, for a range of temperatures. d) The absolute value of the $J_{\mathrm{sc}}$ of the three wavelengths as a function of temperature. The schematic diagrams illustrate the difference in device operation when light absorption occurs in the metal, yielding a gradient in the IV characteristic in reverse bias which is related to electron temperature in the metal absorber, as demonstrated in a) and for low temperatures in b), compared with device operation when light absorption occurs in the semiconductor, yielding a saturating current in reverse bias, as demonstrated in c) and for higher temperatures in b).

The resulting IV characteristics, displayed in Figure 3a, highlight the gradient is indeed determined by the temperature, due to its dependence on the electron Fermi distribution in the metal. The switchover between these two modes of operation is observed in Figure 3b, in which the GaAs can absorb $850 \mathrm{~nm}$ light only above $250 \mathrm{~K}$, resulting in a gradual transition of the IV characteristic. As the temperature increases, the reduction in the gradient of the reverse bias region, shown in Figure $3 \mathrm{a}$, is indicative of the intended mode of operation and is due to the reduction in photo-excited carrier temperature in the metal region. A lower substrate temperature means that for the same additional energy input a lower carrier temperature is reached.

The raw data in Figure $3 \mathrm{~d}$ shows how the $\mathrm{J}_{\text {sc }}$ changes as a function of either metal or semiconductor temperature. When only the metal electron temperature is raised $(890 \mathrm{~nm}$ illumination), the $\mathrm{J}_{\mathrm{sc}}$ increases exponentially, caused by an increasing temperature yielding exponentially more filled electron states at energies where they can be transmitted over the Schottky barrier. For the case where the semiconductor temperature is raised (785nm illumination), the approximate increase in $\mathrm{J}_{\mathrm{sc}}$ follows $\mathrm{T}^{3 / 2}$. This is because the biggest effect of the increase in temperature of the moderately doped semiconductor on the extraction of carriers from the metal is to increase the density of available states to which electrons can transfer, with the density of states dependent on temperature according to $\mathrm{T}^{3 / 2}$. For $850 \mathrm{~nm}$ illumination, a crossover from one regime to the other is observed.

To ensure that results are not caused by direct lattice heating, the IV characteristic as a function of light intensity was recorded to observe the expected linearity of the $V_{o c}$ vs $\ln \left(\mathrm{J}_{\mathrm{sc}}\right)$ response. Figure 4 a shows the light IV characteristic of SB1 at a lattice temperature of $243 \mathrm{~K}$ under illumination at a wavelength of $890 \mathrm{~nm}$ with a laser power of $30-150 \mathrm{~mW}$ (focused to provide intensities between $16-80 \mathrm{~W} / \mathrm{cm} 2$ ).

The reverse bias IV shows a small decrease in its gradient as the power is increased from $30-150 \mathrm{~mW}$ in line with expectations if increasing the illumination power results in an increase in electron temperature. The total decrease in gradient over this range is consistent with an increase in electron temperature of $10 \mathrm{~K}$ according to our modelling. This agrees with the degree of heating caused by a given laser pulse calculated via the electronic heat capacity, as per the method of Groeneveld ${ }^{24}$, with the exception being that for algebraic simplicity we assume that the result is a fully thermalized Fermi distribution so that this temperature can be input to our model.

To determine whether this increase in temperature is a global increase in lattice temperature, or local to the electrons in the thin metallic region, we compared the Jsc and Voc for each illumination power.

Our device results, extracted from Figure 4a, are shown as the black crosses on Figure $4 \mathrm{~b}$ along with two different fits. The black solid line shows a linear relationship between $V_{o c}$ and $\mathrm{J}_{\mathrm{sc}}$ assuming a constant lattice temperature of $243 \mathrm{~K}$. The red solid line shows the modelled effect of increasing the lattice temperature in line with our findings from Figure 4a, which suggests an increase in electron temperature of $10 \mathrm{~K}$ from $30 \mathrm{~mW}-150 \mathrm{~mW}$ excitation.

No sub-linear trend is observed in Figure $4 \mathrm{~b}$, therefore no lattice heating greater than $1 \mathrm{~K}$ occurs in this cell as the laser illumination is increased from $30 \mathrm{~mW}-150 \mathrm{~mW}$. This result, combined with the observation of a decrease in reverse bias gradient in Figure 4a, caused by an increase in electron temperature, is further evidence that we are seeing hot carrier extraction from the thin metallic layer. A non-linear change in $\mathrm{V}_{\text {oc }} \mathrm{vs} \ln \left(\mathrm{J}_{\mathrm{sc}}\right)$ starts to occur for lattice temperatures above 
$263 \mathrm{~K}$ for this cell, so for this reason we only present results in this work up to this temperature.
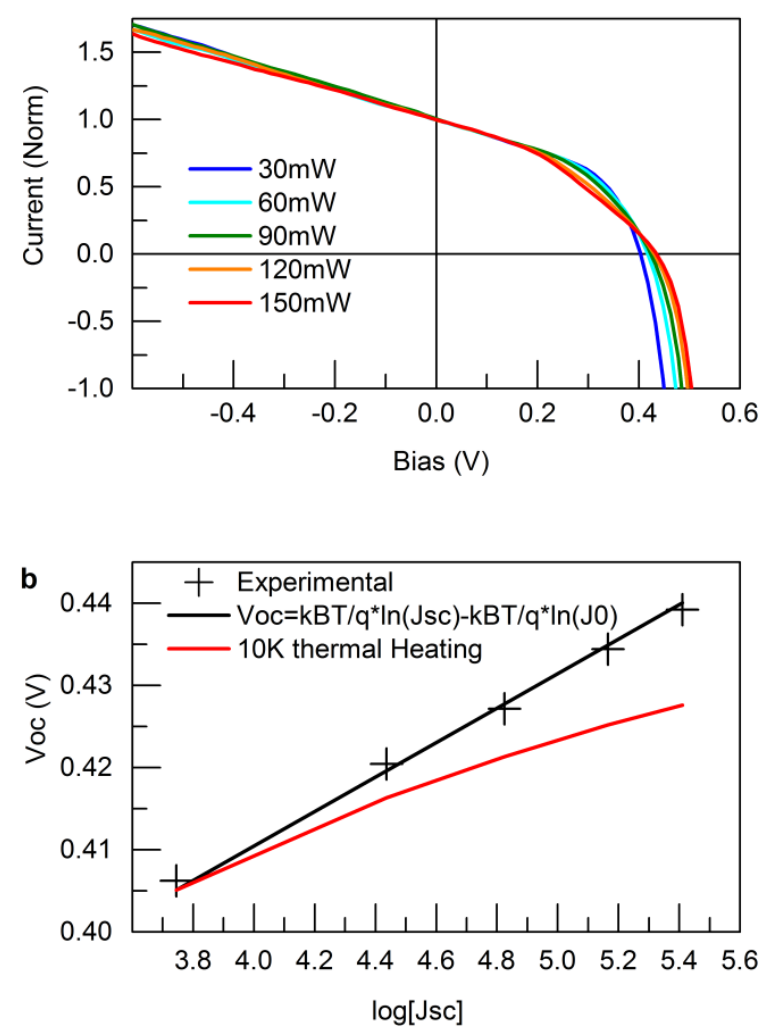

Figure 4| a) The light IV characteristic of SB1 under illumination at $890 \mathrm{~nm}$ and $243 \mathrm{~K}$ for incident laser powers from $30-150 \mathrm{~mW}$, as in the legend. b) $\operatorname{Ln}\left(\mathrm{J}_{\mathrm{sc}}\right)$ vs $\mathrm{V}_{\mathrm{oc}}$ for a $\mathrm{Cr} / \mathrm{GaAs}$ metallic cell under illumination at $890 \mathrm{~nm}$ and $243 \mathrm{~K}$ for incident laser powers from 30-150mW
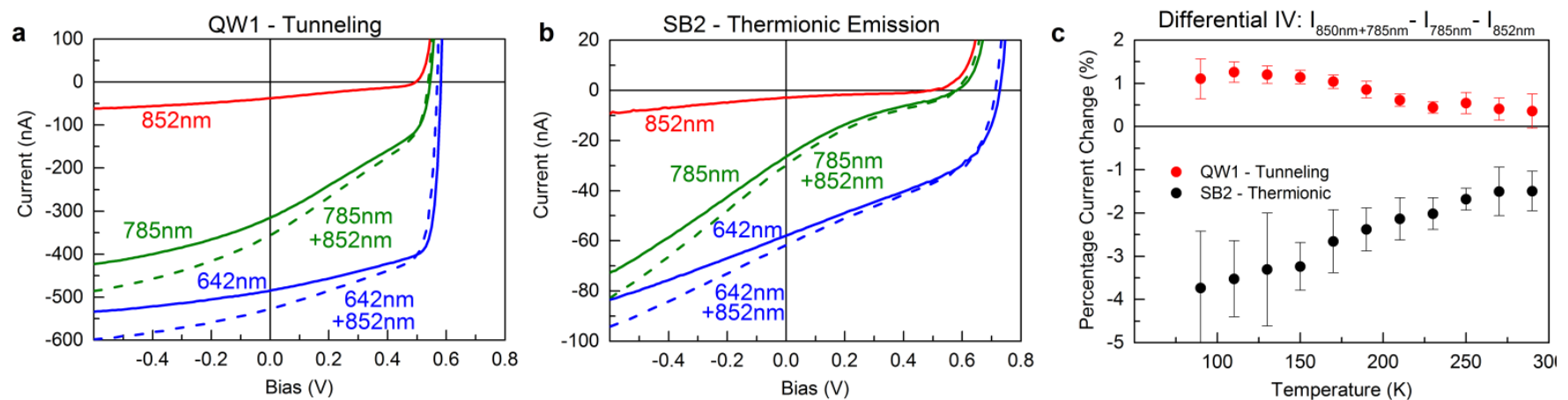

Tunneling Extraction Operation

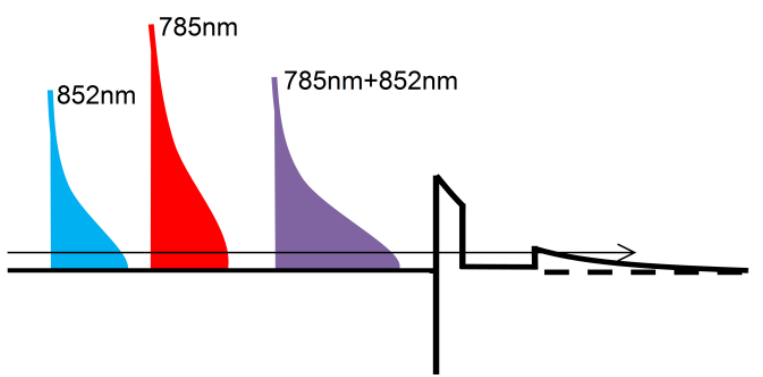

Thermionic Emission Operation

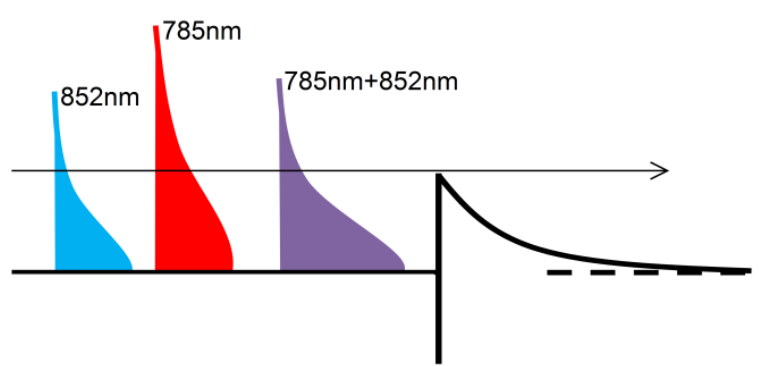

Figure 5 | IV characteristics at 90K for a) QW1 (Tunneling) and b) SB2 (Thermionic Emission) for five different wavelength combinations. c) Differential IV characteristic, subtracting the current at Voc- $0.2 \mathrm{~V}$ generated by individual beams at $785 \mathrm{~nm}$ and $850 \mathrm{~nm}$ from the current generated by illumination with the combination of both beams. The non-zero differential IV gives evidence of electron interaction before extraction: QW1 shows an increase in current from dual beam illumination because the electron population at the tunneling resonance is increased, whereas SB2 shows a decrease in current because the number of electrons in excess of the Schottky barrier height is reduced on dual beam illumination. Schematic illustrations of the electron populations under single and dual-beam illumination are shown for each device structure to illustrate this point. 


\section{Metallic Solar Cells with Tunneling}

To further extend this concept, the ultimate test is to extract carriers photogenerated in metal through tunneling; figuratively combining previous work $^{3,4}$ in which hot electrons were extracted from a semiconductor region by resonant tunneling, with the promise of higher absorption in a thin metallic film. The benefit of selective extraction being a significantly increased limiting efficiency $(85 \%)^{25}$ in comparison to semi-selective extraction due to a lower heat loss. This combination is realised in SB2, in which an $\mathrm{AlGaAs} / \mathrm{GaAs}$ quantum well is used as the energy selective contact to remove hot electrons from a $\mathrm{Cr}$ region by tunneling. QW1 is the comparison cell, for which extraction of electrons is over a Schottky barrier.

Figure $4 \mathrm{a}$ and figure $4 \mathrm{~b}$ show the IV characteristics of QW1 and SB2 under a single beam and dual beam illumination. To determine whether the increase in current observed under dual illumination is linear or non-linear, the current of each single beam illuminated IVs is subtracted from the dual beam $\mathrm{IV}$, and the result divided by the value of the current of the combined beams. This yields a positive value when the current under dual beam illumination shows an increase over the linear addition of the two single beam illuminations.

Figure $4 \mathrm{c}$ shows the relative percentage increase/decrease in the combined illumination current for QW1 and SB2 as a function of temperature. From the differential IV characteristic, it is clear that there is some interaction between the electrons generated by the $785 \mathrm{~nm}$ illumination and the $852 \mathrm{~nm}$ illumination, again supporting the hot carrier mechanism rather than the IPE mechanism.

It is interesting to compare the impact of dual beam illumination on the cell operating by thermionic emission (SB2) and operating by extraction through tunneling (QW1). The introduction of the secondary beam results in a nonlinear increase in current for extraction by tunneling but a decrease for extraction by thermionic emission. The decrease observed for SB2 is due to $852 \mathrm{~nm}$ illumination being only just sufficient to excite carriers to an energy in excess of the barrier height, as seen from the significantly lower current density, for the same illumination intensity, observed from single beam illumination with this wavelength in Figure $4 \mathrm{~b}$. Reducing the average energy of the electron population generated by the combination of the $852 \mathrm{~nm}$ and $785 \mathrm{~nm}$ illumination reduces the number of carriers that exceed the Schottky barrier, giving a negative differential current.

For QW1, with resonant tunnel transport, the opposite effect is observed. This is because shifting the average energy of the electron population lower results in moving the peak of the electron distribution closer to the resonant energy of the well. The resonant energy is calculated as $0.47 \mathrm{eV}$ above the Fermi-level of the metal at zero applied bias, and the full bandstructure of this device is available in the supplementary information. Therefore, while the average energy has been decreased, the total density of electrons available at the transport energy has increased, hence the current has been increased. Importantly, the $\mathrm{V}_{\text {oc }}$ changes by less than the change in the effective barrier height (e.g., from Figure $4 \mathrm{a}$, at $90 \mathrm{~K}$ and $642 \mathrm{~nm}$ illumination $\mathrm{QW1}$ : $\mathrm{V}_{\mathrm{oc}}=0.59 \mathrm{~V}$, SB2: $V_{\text {oc }}=0.74 \mathrm{~V}$ giving a $\mathrm{V}_{\text {oc }}$ reduction of $0.15 \mathrm{~V}$ compared to the effective barrier height reduction of $0.2 \mathrm{~V}$ ). This is not simply a trade-off between $\mathrm{J}_{\mathrm{sc}}$ and $\mathrm{V}_{\mathrm{oc}}$ between these cells, as present in conventional photovoltaic cells.

To further investigate this effect, QW1 and SB2 were illuminated with $1550 \mathrm{~nm}$ light in addition to $642 \mathrm{~nm}$ light to observe whether any increase in the photo-response is seen when the additional wavelength itself $(1550 \mathrm{~nm})$ has no photo-response. Figure 5 shows that the dark current (black) and current under illumination with $1550 \mathrm{~nm}$ (red dash) is identical over the range of voltages at $90 \mathrm{~K}$ for both QW1 and SB2, showing that it is not sufficient to generate current on its own. However, adding illumination at $1550 \mathrm{~nm}$ to a beam at $642 \mathrm{~nm}$ shows that an additive photo-response (blue line) is observed for the case of QW1, but not for the case of SB2. While the magnitude of the effect is small (adding 1550nm illumination increases the photocurrent by $1 \%$ relative to the $642 \mathrm{~nm}$ illumination on its own), this is an important proof of principle, showing that this cell is capable of producing a photocurrent from light with a wavelength that, on its own, is at too low an energy to generate a photocurrent.

a
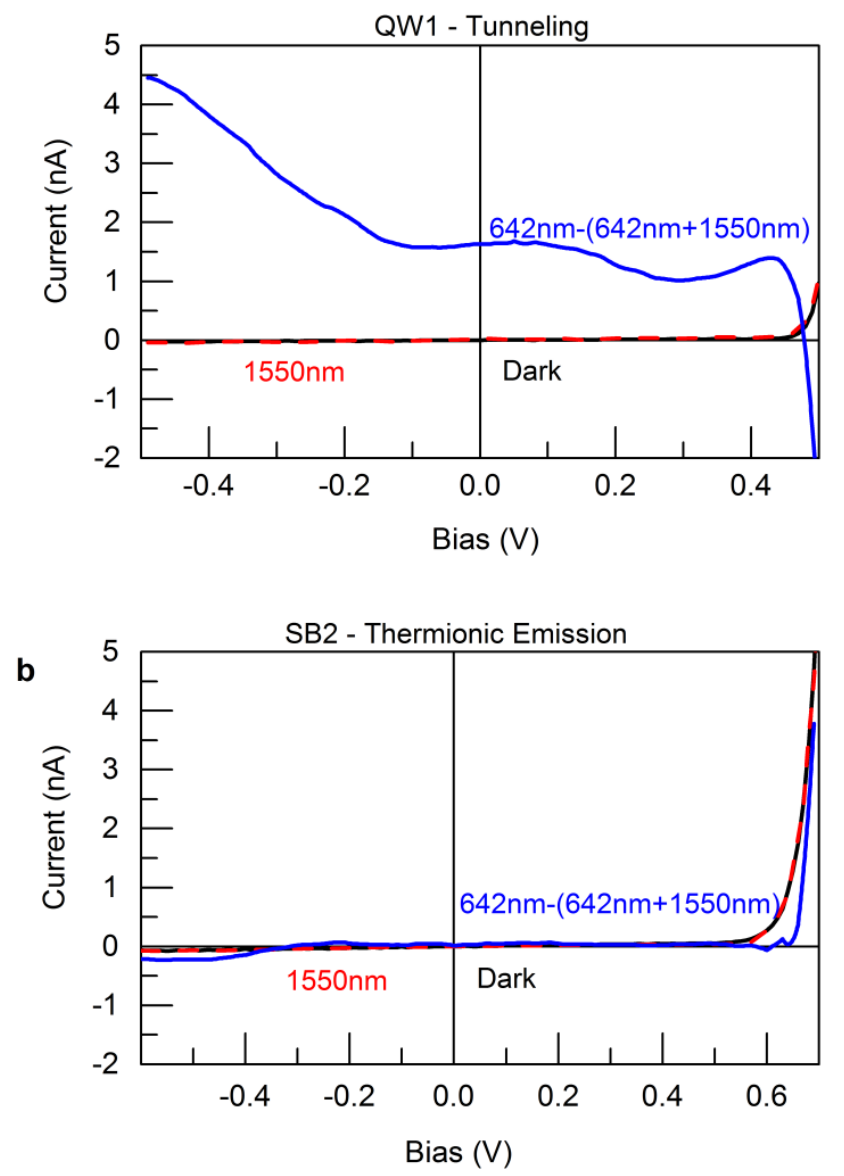

Figure 6 | The IV characteristics at 90K of QW1 (tunneling extraction) (a) and SB2 (thermionic extraction) (b) under single wavelength illumination at $1550 \mathrm{~nm}$, demonstrating no observable photocurrent (red) in comparison with the dark current (black) for either device. Whereas a differential increase in current with dual beam illumination of $642 \mathrm{~nm}+1550 \mathrm{~nm}$ is observed for QW1 but not SB2, demonstrating that electron interaction occurs before electron extraction and the observation of a current generated by illumination with light which, on its own, would generate no photocurrent.

QW1 can use long wavelength light to generate a photocurrent when used in conjunction with a shorter wavelength illumination precisely because of the redistribution of energy among the excited carriers. SB2 suffers no reduction in the photocurrent until a reverse bias of $0.4 \mathrm{~V}$ is applied, because $642 \mathrm{~nm}$ illumination is sufficiently in excess of the Schottky barrier for the $1550 \mathrm{~nm}$ illumination not to reduce the average electron energy below the energy threshold for extraction. 


\section{Conclusions}

The metallic solar cell has various advantages over a conventional photovoltaic cell, in particular, the promise of using the full solar spectrum and achieving absorption in an ultra-thin layer. We have shown two different mechanisms taking advantage of the absorption of light in a thin metal layer, through thermionic emission and tunneling from the metal layer into an adjacent semiconductor region. In these structures, the IV characteristics show that redistribution of energy occurs in the electron population, an essential feature of a hot carrier cell, and furthermore that the result of this redistribution is a cell which shows a photocurrent from what would normally be deemed sub-band-gap illumination.

Further improvements to the absorption of light in such thin metallic films are straightforward. In particular, building on work using a metallic absorber in a dielectric cavity ${ }^{26}$, the reflection from the top surface could be reduced and a resonant cavity created in what is effectively an optical frequency Jaumann layer ${ }^{27}$.

A metallic structure could be used in tandem with existing photovoltaic cells and a standard process, replacing or enhancing existing metallisations. The tunneling structures could also be exploited in photodetectors sensitive to very broad ranges of wavelengths, since they show significantly increased current over a standard Schottky barrier and could in principle operate in wavelength ranges only limited by the relative potentials of the Fermi energy in the metal and the resonant energy of the quantum well, rather than being limited by the Schottky barrier height. Additionally, the characteristic IV profile offers some discrimination of the photon energy absorbed by the device ${ }^{28}$.

The data presented above and the range of potential applications for this structure suggest that the metallic photovoltaic device could find application well beyond the confines of photovoltaic solar power conversion.

\section{References}

1. Ross, R. T. \& Nozik, A. J. Efficiency of hot-carrier solar energy converters. J. Appl. Phys. 53, 3813-3818 (1982).

2. Yagi, S., Hijikata, Y., Okada, Y. \& Yaguchi, H. Quantum well double barrier resonant tunneling structures for selective contacts of hot carrier solar cells. in Proceedings of the 37th IEEE Photovoltaic Specialists Conference 2011, Hawaii, USA 1213-1217 (2011).

3. Dimmock, J. A. R., Day, S., Kauer, M., Smith, K. \& Heffernan, J. Demonstration of a hot-carrier photovoltaic cell. Prog. Photovolt. Res. Appl. 22, 151-160 (2014).

4. Dimmock, J. A. R. et al. Optoelectronic characterization of carrier extraction in a hot carrier photovoltaic cell structure. J. Opt. 18, 074003 (2016).

5. Hirst, L. C., Fujii, H., Wang, Y., Sugiyama, M. \& EkinsDaukes, N. J. Hot Carriers in Quantum Wells for Photovoltaic Efficiency Enhancement. IEEE J. Photovolt. 4, 244-252 (2014).

6. Le Bris, A. et al. Thermalisation rate study of GaSb-based heterostructures by continuous wave photoluminescence and their potential as hot carrier solar cell absorbers. Energy Environ. Sci. 5, 6225 (2012).
7. Esmaielpour, H. et al. Suppression of phonon-mediated hot carrier relaxation in type-II InAs/AlAsxSb1 - x quantum wells: a practical route to hot carrier solar cells. Prog. Photovolt. Res. Appl. 24, 591-599 (2016).

8. Conibeer, G. et al. Hot carrier solar cell absorber prerequisites and candidate material systems. Sol. Energy Mater. Sol. Cells 135, 124-129 (2015).

9. Shrestha, S. K. et al. Evaluation of Hafnium Nitride and Zirconium Nitride as Hot Carrier Absorber. in Proceedings of the 40th IEEE Photovoltaic Specialists Conference (2014).

10.Nguyen, D.-T. et al. Quantitative experimental assessment of hot carrier-enhanced solar cells at room temperature. Nat. Energy 3, 236-242 (2018).

11. Rosenwaks, Y. et al. Hot-carrier cooling in GaAs: Quantum wells versus bulk. Phys. Rev. B 48, 1467514678 (1993).

12. White, T. P. \& Catchpole, K. R. Plasmon-enhanced internal photoemission for photovoltaics: Theoretical efficiency limits. Appl. Phys. Lett. 101, 073905 (2012).

13. Chang, Y.-J. \& Shih, K.-H. Solar energy conversion via internal photoemission in aluminum, copper, and silver: Band structure effects and theoretical efficiency estimates. J. Appl. Phys. 119, 183101 (2016).

14.Leenheer, A. J., Narang, P., Lewis, N. S. \& Atwater, H. A. Solar energy conversion via hot electron internal photoemission in metallic nanostructures: Efficiency estimates. J. Appl. Phys. 115, 134301 (2014).

15. McFarland, E. W. \& Tang, J. A photovoltaic device structure based on internal electron emission. Nature 421, 616-618 (2003).

16. Russell, K. J. et al. Room-temperature electro-optic upconversion via internal photoemission. Appl. Phys. Lett. 82, 2960 (2003).

17. Takeda, Y. Requisites for Highly Efficient Hot-Carrier Solar Cells. in Quantum Dot Solar Cells (eds. Wu, J. \& Wang, Z. M.) 15, 187-232 (Springer New York, 2014).

18.Dimmock, J. A. R., Kauer, M., Stavrinou, P. N. \& EkinsDaukes, N. J. A metallic hot carrier photovoltaic cell. in Proc. SPIE 9358, Physics, Simulation, and Photonic Engineering of Photovoltaic Devices IV (eds. Freundlich, A., Guillemoles, J.-F. \& Sugiyama, M.) 935810 (2015). doi:10.1117/12.2077573

19. Khurgin, J. B. How to deal with the loss in plasmonics and metamaterials. Nat. Nanotechnol. 10, 2-6 (2015).

20.Clavero, C. Plasmon-induced hot-electron generation at nanoparticle/metal-oxide interfaces for photovoltaic and photocatalytic devices. Nat. Photonics 8, 95-103 (2014).

21. Maier, S. A. \& Atwater, H. A. Plasmonics: Localization and guiding of electromagnetic energy in metal/dielectric structures. J. Appl. Phys. 98, 011101 (2005). 
22.Pilkington, S. J., Missous, M. \& Woolf, D. A. On the Richardson constant of intimate metal-GaAs (111)B Schottky diodes grown by molecular beam epitaxy. $J$. Appl. Phys. 74, 6256-6260 (1993).

23. Cho, S.-H. et al. A black metal-dielectric thin film for high-contrast displays. J. Korean Phys. Soc. 55, 501-507 (2009).

24.Groeneveld, R. H. M., Sprik, R. \& Lagendijk, A. Femtosecond spectroscopy of electron-electron and electron-phonon energy relaxation in $\mathrm{Ag}$ and $\mathrm{Au}$. Phys. Rev. B 51, 11433-11445 (1995).

25. Würfel, P., Brown, A. S., Humphrey, T. E. \& Green, M. A. Particle conservation in the hot-carrier solar cell. Prog. Photovolt. Res. Appl. 13, 277-285 (2005).

26. Hilsum, C. Infrared Absorption of Thin Metal Films. $J$. Opt. Soc. Am. 44, 188-188 (1954).

27.Ke, L., Xin, Z., Xinyu, H. \& Peng, Z. Analysis and design of multilayer Jaumann absorbers. in 2011 IEEE International Conference on Microwave Technology Computational Electromagnetics (ICMTCE) 81-84 (2011). doi:10.1109/ICMTCE.2011.5915168

28.Dimmock, J. A. R., Kauer, M., Ekins-Daukes, N. J. \& Stavrinou, P. N. Energy selective photodetector. (2017).

\section{Acknowledgments}

J. Dimmock gratefully acknowledges a Royal Commission for the Exhibition of 1851 Industrial Fellowship. 